Received March 14, 2019

Revised May 20, 2019

Accepted June 3, 2019

\author{
Corresponding author \\ Jung-Won Hwang, M.D., Ph.D. \\ Department of Anesthesiology \\ and Pain Medicine, Seoul National \\ University Bundang Hospital, 82 \\ Gumi-ro 173beon-gil, Bundang-gu, \\ Seongnam 13620, Korea \\ Tel: 82-31-787-7499 \\ Fax: 82-31-787-4063 \\ E-mail: jungwon@snubh.org \\ ORCID \\ https://orcid.org/0000-0002-0887-6889
}

\section{Association of preoperative pain in knee and external to knee with postoperative pain outcome after total knee arthroplasty}

\author{
Tak Kyu Oh${ }^{1}$, Chong Bum Chang ${ }^{2}$, Hyun-Jung Shin', Sunghee Han ${ }^{1,3}$, \\ Sang-Hwan Do ${ }^{1,3}$, Hyun Hee Cho', and Jung-Won Hwang ${ }^{1,3}$
}

'Department of Anesthesiology and Pain Medicine, Seoul National University Bundang Hospital, Seongnam, Departments of ${ }^{2}$ Orthopedic Surgery, ${ }^{3}$ Anesthesiology and Pain Medicine, Seoul National University College of Medicine, Seoul, Korea

Background: Preoperative pain in the symptomatic knee may predict postoperative pain severity in patients undergoing total knee arthroplasty (TKA). However, the effect of preoperative pain external to the knee on postoperative pain is unclear. This study evaluated postoperative pain outcomes in TKA patients according to the presence of preoperative pain in the knee only or in the knee and external to the knee.

Methods: We retrospectively assessed medical records of patients who underwent unilateral TKA. The relationship between reported preoperative pain characteristics and morphine equivalent consumption or numerical rating scale (NRS) pain scores on postoperative day (POD) 0-3 was assessed using a multivariable generalized linear model.

Results: In total, 3,429 adult patients who underwent their first TKA were included; $2,864(83.5 \%)$ patients preoperatively experienced only knee pain and $565(16.5 \%)$ knee pain with external to knee pain. Preoperative pain in the knee and external to the knee was associated with 5\% higher morphine equivalent consumption on POD 0-3 compared to preoperative knee pain only (exponentiated regression coefficient: 1.05; 95\% confidence interval: 1.02 to $1.09 ; P=0.004)$. However, the NRS pain scores on POD $0,1,2$, and 3 and adjuvant analgesics consumption (acetaminophen and ketorolac) on POD 0-3 were not significantly different between the two groups $(P>0.05)$.

Conclusions: This study showed that there was an increase of morphine equivalent consumption during POD 0-3 in patients with preoperative knee pain with external to knee pain than in patients with preoperative only knee pain.

Keywords: Analgesics, opioid; Arthralgia; Pain, postoperative.

\section{INTRODUCTION}

Total knee arthroplasty (TKA) is one of the most performed surgical procedures in the United States [1]. The primary purposes of TKA include pain relief, and improvement in mobility and health-related quality of life in patients with advanced knee osteoarthritis [2]. Among the patients who undergo
TKA, 20\% are reported to experience persistent postsurgical pain [3], and high pain intensity in the acute postoperative period is a high predictor for chronic postoperative pain [4]. Therefore, an understanding of acute postoperative pain is important from a clinical perspective, i.e., it is crucial to understand the conditions under which severe postoperative pain occurs.

This is an Open Access article distributed under the terms of the Creative Commons Attribution Non-Commercial License (http://creativecommons.org/licenses/by-nc/4.0) which permits unrestricted non-commercial use, distribution, and reproduction in any medium, provided the original work is properly cited. 
Considering that severe osteoarthritis (OA) - one of the most frequent indications of TKA-occurs frequently in older female patients [5], patients who are scheduled for TKA likely have other concomitant illnesses, such as fibromyalgia or other musculoskeletal conditions. In such painful musculoskeletal conditions, increased pain sensitivity in response to a variety of pain stimuli has been reported in patients with OA at sites both over and away from symptomatic joints [6,7]. Widespread pain or hyperalgesia has been found in some severe knee OA patients, and this is most likely due to impaired pain inhibition, which suggests "central sensitization" [8]. Central sensitization at the level of the dorsal horn includes temporal and spatial summation, and is characterized by an increased pain response to repeated stimulation or to a larger area of stimulation, respectively [9]. Among these summations, spatial summation from various stimuli is known as an important factor for developing central sensitization in patients with fibromyalgia [10]. From this perspective, preoperative musculoskeletal chronic pain, not caused by a symptomatic knee joint, could affect postoperative pain after TKA by spatial summation; however, information regarding this issue is still lacking.

Therefore, this study aimed to assess the effects of preoperative chronic pain-divided into knee pain only and knee pain with external to knee pain-on the pain outcome after TKA under spinal anesthesia.

\section{MATERIALS AND METHODS}

This study was a retrospective observational study and was performed with the Institutional Review Board (IRB) approval (no. B-1711/432-103). The requirement for informed consent was waived by the IRB due to the retrospective nature of the study. The study was based on the medical records of adult patients who were 20 years or older and were admitted between January 2005 and October 2017 for elective TKA under spinal anesthesia. The following cases were excluded from the analysis: those with incomplete medical records regarding opioid usage or pain score; those with reoperation within 3 days of the original operation; or those who underwent a second TKA, which reportedly causes more severe pain in the patients [11].

\section{Perioperative care for patients undergoing TKA under spinal anesthesia}

Anesthesiologists administered spinal anesthesia with hyperbaric local anesthetics (Marcaine Spinal 0.5\% Heavy, Astra Zeneca, Sweden) and fentanyl 10-15 $\mu$ g for unilateral TKA. The objective block level was T10, and after confirming the appropriateness of the block level, sedation was performed during the surgery using propofol or dexmedetomidine. Postoperative pain management was performed using fentanylbased intravenous (IV) patient-controlled analgesia (PCA). The regimen, based on the patient's weight and underlying diseases, was as follows: fentanyl 8-15 $\mu \mathrm{g} \mathrm{ml}^{-1}, 1 \mathrm{ml}$ bolus dose without background infusion, and a 10-min lockout time. IV PCA (100 ml total) was mostly used on postoperative day (POD) 0-1, and additional opioid analgesics (e.g., morphine, fentanyl, oxycodone) were used after IV PCA, as per the patient's request or the surgeon's decision. For adjuvant pain control, either periarticular injection was given by the surgeon during surgery, or femoral nerve block was administered by the anesthesiologist. While $0.375 \%$ ropivacaine with morphine was used for intraoperative periarticular injection, $0.2 \%$ ropivacaine was infused through a catheter for femoral nerve block, at a rate of $4 \mathrm{ml}$ per hour, from the start of the surgery until $48 \mathrm{~h}$ after the operation. To avoid the effects of local anesthetic toxicity, the attending physician first aspirated through the needle before injection to avoid blood injection, and injected a small test dose (1-2 ml) to check for immediate toxicity before injecting the full dose.

\section{Pain reporting system}

The location and intensity of pain at all sites experienced by the patients 1-2 days prior to TKA surgery are routinely recorded at our institution. The pain can be classified as a mixed-type pain, which involves somatic and neuropathic pain. The information on location of pain includes all the body sites at which the patient experienced pain at admission. Pain intensity was represented using the numeric rating scale (NRS) score, ranging from 0 to 10 . The patients were divided into two groups (based on the pain location as only knee pain and knee pain with external to knee pain) according to the preoperative pain reports. Postoperative pain intensity was also checked using the NRS scores during rest and 
during movement, and all checks were regularly performed once every $4-6 \mathrm{~h}$ by registered nurses.

\section{Measurement}

Data collected for the study included demographic information (age, sex, and body mass index), American Society of Anesthesiologists physical status, Charlson comorbidity index [12], intraoperative sedative uses (propofol or dexmedetomidine), preoperative analgesics use (opioid analgesics and non-opioid analgesics) and postoperative pain outcome for POD 0-3 (morphine equivalent consumption, NRS pain scores, and adjuvant analgesics use [acetaminophen and ketorolac]). Preoperative non-opioid analgesics did not include anticonvulsants or antidepressants, and generally, all preoperative regular medications were re-started after surgery at POD 0. The postoperative NRS pain scores during rest and movement were represented using an average daily value of the multiple assessments made on each day, and morphine equivalent consumption was derived by converting all intravenous and oral opioid analgesics used by the patients into their morphine equivalents (mg), according to the standard conversion ratio [13] (Table 1). All medical records were collected by medical record technicians who were blinded to the purpose of the study. The main researchers of the study were blinded to the patient characteristics until after the statistical analyses had been completed.

\section{Statistical analysis}

The baseline characteristics of the patients were represented using mean with standard deviation (SD) and number with percentage. In order to assess how each variable affects the morphine equivalent consumption for POD 0-3, we performed a generalized linear regression analysis. In the univariable generalized linear model (GLM), the covariates with $\mathrm{P}<0.2$ for our two main independent variables (preoperative pain and analgesics use) were then selected for further analysis using a multivariable GLM.

Next, we performed 10 multivariable GLMs according to the preoperative pain site for 10 dependent variables: postoperative NRS 0, 1, 2, and 3, and adjuvant analgesic consumption (acetaminophen and ketorolac) after TKA. The backward variable selection method with an elimination criterion of 0.05 was used to fit the final multivariable model for these 10 dependent variables. For the GLMs, gamma distribution was assumed and the log link function was used for all dependent

Table 1. Equianalgesic Opioid Conversion Table

\begin{tabular}{llc}
\hline Opioid & $\begin{array}{c}\text { Administration } \\
\text { route }\end{array}$ & $\begin{array}{c}\text { Dose equivalent to 10 mg } \\
\text { of oral morphine (mg) }\end{array}$ \\
\hline Morphine & Oral & 10 \\
Morphine & Intravenous & 3.3 \\
Hydromorphone & Oral & 2 \\
Fentanyl & Intravenous & 0.03 \\
Oxycodone & Oral & 7 \\
Codeine & Oral & 80 \\
Tramadol & Oral & 40
\end{tabular}

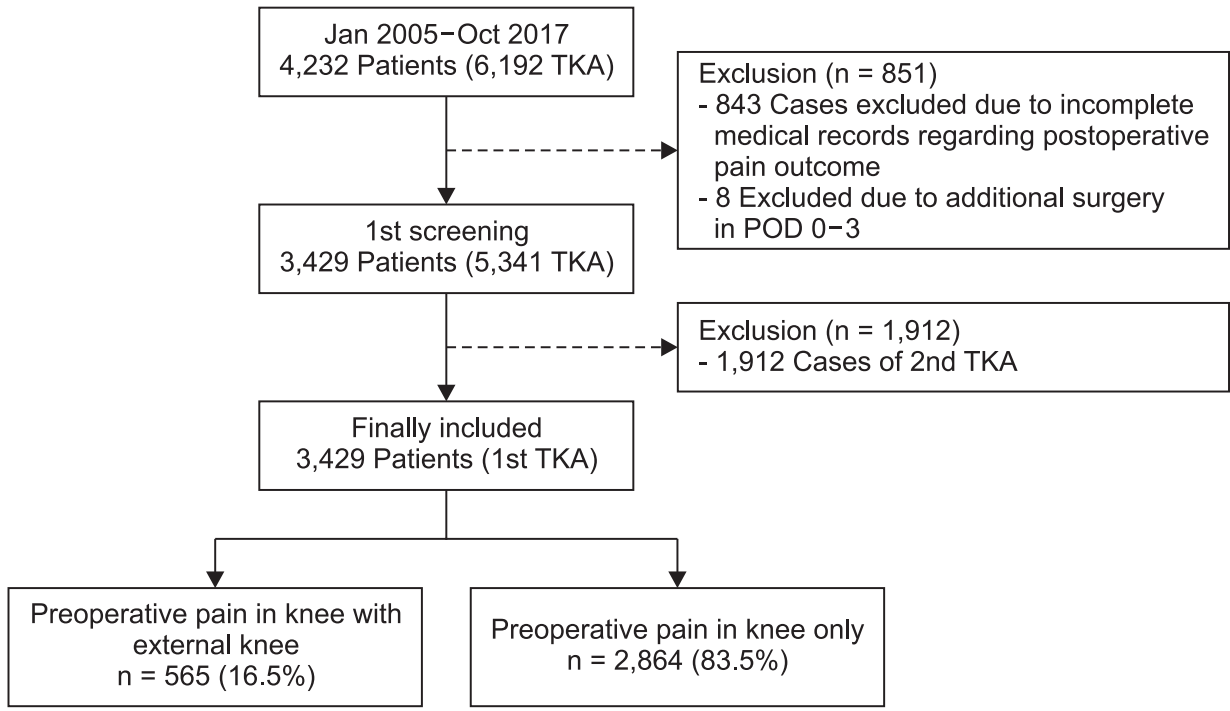

Fig. 1. Flow chart for patient selection. TKA: total knee arthroplasty, POD: postoperative day. 
variables (morphine equivalent consumption, postoperative NRS $0,1,2$, and 3, and adjuvant analgesic consumption after TKA). The results of GLM were presented as exponentiated regression coefficient (Exp. Coef) with 95\% confidence interval (CI). IBM SPSS version 24.0 (IBM Corp., USA) was used for all analyses, with the statistical significance set at $\mathrm{P}<0.05$.

\section{RESULTS}

Between January 2005 and October 2017, 4,232 patients were admitted for TKA and 6,192 unilateral TKA procedures were performed under spinal anesthesia. The following cases were excluded from the study: 843 were excluded due to incomplete medical records on pain outcome; 8 were excluded due to complication-related additional surgery on POD 0-3; and 1,912 cases were excluded because the surgery involved a second TKA. The final cohort included 3,429 adult (aged $\geq 20$ years) patients who underwent their first TKA (Fig. 1). The baseline characteristics of these patients are outlined in Table 2. Among the entire cohort, 2,864 (83.5\%) patients experienced preoperative only knee pain with a mean NRS \pm SD of $4.7 \pm 2.1$, while 565 (16.5\%) patients experienced knee pain along with external to knee pain with a mean NRS \pm SD of $5.2 \pm 2.1$. Furthermore, 1,383 (40.3\%) patients were receiving regular analgesics for pain control and $50(1.5 \%)$ patients were using opioid analgesics.

\section{Morphine equivalent consumption during postoperative day 0-3}

Table 3 shows the results of the univariable GLM for the morphine equivalent consumption on POD 0-3. The results of the multivariable GLM using the variables with $\mathrm{P}<0.2$ from the univariable GLM are presented in Table 4 . The results of the final multivariable GLM are shown in Table 4. Morphine equivalent consumption on POD $0-3$ was significantly $5 \%$ higher in the group with preoperative knee pain with external to knee pain, compared to the patients who reported preoperative knee pain only (Exp Coef: 1.05; 95\% CI, 1.02 to 1.09; P $=0.004$ ). Additionally, the morphine equivalent consumption of patients in the non-opioid analgesic use group and opioid analgesic use group was not significantly different from that of patients in the "no preoperative regular analgesics use" group $(\mathrm{P}>0.05)$. Among other variables, femoral nerve block
Table 2. Patient Characteristics $(n=3,429)$

\begin{tabular}{|c|c|}
\hline Characteristic & Value \\
\hline Age (yr) & $70.7 \pm 6.9$ \\
\hline Body mass index $\left(\mathrm{kg} / \mathrm{m}^{2}\right)$ & $28.0 \pm 4.6$ \\
\hline Sex (male) & $318(9.3)$ \\
\hline \multicolumn{2}{|l|}{ ASA classification } \\
\hline 1 & $420(12.2)$ \\
\hline 2 & $2,806(81.8)$ \\
\hline$\geq 3$ & $203(5.9)$ \\
\hline \multicolumn{2}{|l|}{ Charlson comorbidity index } \\
\hline$\leq 2$ & $1.0 \pm 0.9$ \\
\hline$>2$ & $186(5.4)$ \\
\hline Surgery time (min) & $109.4 \pm 26.1$ \\
\hline Intraoperative propofol use & $1,038(30.3)$ \\
\hline Intraoperative dexmedetomidine use & $250(7.3)$ \\
\hline \multicolumn{2}{|l|}{ Preoperative pain reports } \\
\hline Pain in knee only & $2,864(83.5)$ \\
\hline NRS & $4.7 \pm 2.1$ \\
\hline Knee pain + external knee pain & $565(16.5)$ \\
\hline NRS & $5.2 \pm 2.1$ \\
\hline Regular non-opioid analgesics use & 1,333 (38.9) \\
\hline Regular opioid analgesics use & $50(1.5)$ \\
\hline \multicolumn{2}{|l|}{ Postoperative pain outcome (NRS) } \\
\hline Resting pain POD 0 & $1.9 \pm 1.5$ \\
\hline Resting pain POD 1 & $2.3 \pm 1.3$ \\
\hline Resting pain POD 2 & $2.6 \pm 1.1$ \\
\hline Resting pain POD 3 & $2.7 \pm 1.0$ \\
\hline Moving pain POD 0 & $3.7 \pm 2.0$ \\
\hline Moving pain POD 1 & $3.9 \pm 1.9$ \\
\hline Moving pain POD 2 & $4.2 \pm 1.8$ \\
\hline Moving pain POD 3 & $4.2 \pm 1.8$ \\
\hline \multicolumn{2}{|l|}{ Analgesics consumption in POD 0-3 } \\
\hline Morphine equivalent (mg) & $1,385.7 \pm 508.3$ \\
\hline Acetaminophen (mg) & $2,650.2 \pm 1,188.4$ \\
\hline Ketorolac use (mg) & $122.3 \pm 54.8$ \\
\hline \multicolumn{2}{|l|}{ Adjuvant pain management } \\
\hline Femoral nerve block & 2,377 (69.3) \\
\hline Intraoperative periarticular injection & $3,342(97.5)$ \\
\hline
\end{tabular}

Values are presented as mean \pm SD or number (\%). ASA: American Society of Anesthesiologists, NRS: numeric rating scale, POD: postoperative day.

and intraoperative propofol use were associated, respectively, with a 11\% (Exp. Coef: 0.89; 95\% CI: 0.87 to 0.92; P < 0.001) and 4\% (Exp. Coef: 0.96; 95\% CI: 0.93 to $0.99 ; \mathrm{P}=0.004$ ) lower morphine equivalent consumption during POD 0-3.

\section{NRS pain score on postoperative day $0,1,2,3$ and adjuvant analgesics use}

Table 5 shows the results of the multivariable GLM performed using the NRS pain score on PODs $0,1,2$, and 3, and adjuvant analgesic use (acetaminophen and ketorolac) as 
Table 3. Univariable Generalized Linear Model for Morphine Equivalent (mg) Consumption in POD 0-3 after TKA under Spinal Anesthesia

\begin{tabular}{|c|c|c|}
\hline \multirow{2}{*}{ Variable } & Univariable model & \multirow{2}{*}{$P$ value } \\
\hline & Exp. Coef $(95 \% \mathrm{Cl})$ & \\
\hline Age (yr) & $1.01(1.00,1.01)$ & $<0.001$ \\
\hline Sex, female (vs. male) & $0.99(0.94,1.04)$ & 0.987 \\
\hline Body mass index $\left(\mathrm{kg} / \mathrm{m}^{2}\right)$ & $1.00(1.00,1.00)$ & 0.954 \\
\hline \multicolumn{3}{|l|}{ ASA physical status } \\
\hline 1 & 1 & \\
\hline 2 & $0.95(0.90,0.99)$ & 0.011 \\
\hline$\geq 3$ & $0.97(0.91,1.05)$ & 0.467 \\
\hline Charlson comorbidity index: > 2 (vs. $\leq 2)$ & $1.05(0.99,1.12)$ & 0.103 \\
\hline Surgery time (min) & $1.01(1.00,1.01)$ & 0.875 \\
\hline Intraoperative propofol use & $0.86(0.83,0.89)$ & $<0.001$ \\
\hline Intraoperative dexmedetomidine use & $1.17(1.11,1.24)$ & $<0.001$ \\
\hline \multicolumn{3}{|l|}{ Adjuvant pain management } \\
\hline Femoral nerve block & $0.90(0.87,0.93)$ & $<0.001$ \\
\hline Intraoperative periarticular injection & $0.90(0.82,0.99)$ & 0.026 \\
\hline \multicolumn{3}{|l|}{ Year of surgery } \\
\hline $2005-2008$ & 1 & \\
\hline 2009-2011 & $1.81(1.74,1.88)$ & $<0.001$ \\
\hline 2012-2014 & $1.75(1.69,1.82)$ & $<0.001$ \\
\hline 2015-2017 & $1.94(1.87,2.02)$ & $<0.001$ \\
\hline Knee pain + external knee pain (vs. only knee pain) & $1.02(0.98,1.06)$ & 0.309 \\
\hline \multicolumn{3}{|l|}{ Preoperative analgesics use } \\
\hline No preoperative regular analgesics use & 1 & \\
\hline Preoperative non-opioid analgesics use & $0.81(0.78,0.83)$ & $<0.001$ \\
\hline Preoperative opioid analgesics use & $0.96(0.86,1.08)$ & 0.529 \\
\hline
\end{tabular}

Covariates with $\mathrm{P}<0.2$ (age, ASA physical status, Charlson comorbidity index, adjuvant pain management, and year of surgery) were included in the multivariable GLM in Table 3. POD: postoperative day, TKA: total knee arthroplasty, Exp. Coef: exponentiated regression coefficient, Cl: confidence interval, ASA: American Society of Anesthesiologists, GLM: generalized linear model.

dependent variables, along with backward variable selection using an elimination criterion of 0.05 . No significant association was found between the site of pain (knee only vs. knee pain with external to knee pain) and these factors $(P>0.05)$.

\section{DISCUSSION}

The results of our study showed that patients who experienced pain at sites external to the knee in addition to pain at the knee had increased morphine equivalent consumption during POD 0-3 after TKA under spinal anesthesia, than did patients with preoperative knee pain only. There was no significant association regarding NRS pain scores on PODs 0 , 1,2 , and 3. Our finding is clinically important because it suggests that preoperative widespread pain besides the symptomatic knee is associated with an increase in postoperative pain following TKA.

Chronic OA pain is a known contributing factor for central sensitization [14,15], and Rakel et al. [16] demonstrated that severe preoperative knee pain is associated with increased postoperative pain after TKA, which could be explained as central sensitization before surgery. However, that study did not investigate pain external to the knee before surgery. Patients with external to the knee pain with knee pain may receive more pain stimuli than patients with knee pain only, through a process known as spatial summation [10,17]. Spatial summation, which may be caused by pain at multiple sites, is one of the factors that is related to abnormal endogenous pain modulation in chronic pain conditions [18]. It is reported that endogenous pain inhibitory systems could be activated in patients with widespread pain by spatial summation in fibromyalgia patients [19]. If the pathway of the central nervous system is activated abnormally in chronic pain conditions, it may result in the enhanced pain through central modulation [20]. Although no study has focused on the association between spatial summation in OA patients and postoperative pain outcomes after TKA, a study has reported that spatial summation in chronic OA patients is clini- 
Table 4. Multivariable Generalized Linear Model for Morphine Equivalent (mg) Consumption in POD 0-3 after TKA Under Spinal Anesthesia

\begin{tabular}{|c|c|c|}
\hline \multirow{2}{*}{ Variable } & Multivariable model & \multirow{2}{*}{$P$ value } \\
\hline & Exp. Coef $(95 \% \mathrm{Cl})$ & \\
\hline Age (yr) & $1.00(1.00,1.01)$ & 0.086 \\
\hline \multicolumn{3}{|l|}{ ASA physical status } \\
\hline 1 & 1 & \\
\hline 2 & $0.98(0.94,1.02)$ & 0.236 \\
\hline$\geq 3$ & $1.00(0.94,1.06)$ & 0.935 \\
\hline Charlson comorbidity index: > 2 (vs. $\leq 2)$ & $0.95(0.89,1.00)$ & 0.049 \\
\hline Intraoperative propofol use & $0.96(0.93,0.99)$ & 0.004 \\
\hline Intraoperative dexmedetomidine use & $1.04(0.99,1.09)$ & 0.131 \\
\hline \multicolumn{3}{|l|}{ Adjuvant pain management } \\
\hline Femoral nerve block & $0.89(0.87,0.92)$ & $<0.001$ \\
\hline Intraoperative periarticular injection & $1.06(0.98,1.15)$ & 0.155 \\
\hline \multicolumn{3}{|l|}{ Year of surgery } \\
\hline $2005-2008$ & 1 & \\
\hline 2009-2011 & $1.77(1.70,1.84)$ & $<0.001$ \\
\hline 2012-2014 & $1.72(1.65,1.80)$ & $<0.001$ \\
\hline $2015-2017$ & $1.89(1.81,1.97)$ & $<0.001$ \\
\hline Knee pain + external knee pain (vs. only knee pain) & $1.05(1.02,1.09)$ & 0.004 \\
\hline \multicolumn{3}{|l|}{ Preoperative analgesics use } \\
\hline No preoperative regular analgesics use & 1 & \\
\hline Preoperative non-opioid analgesics use & $0.98(0.95,1.01)$ & 0.146 \\
\hline Preoperative opioid analgesics use & $0.98(0.88,1.08)$ & 0.634 \\
\hline
\end{tabular}

Multivariate model: Covariates with $\mathrm{P}<0.2$ from the univariable model in Table 2 (age, ASA physical status, Charlson comorbidity index, adjuvant pain management, and year of surgery) were included in the multivariable model. Model 1 and Model 2 were made to avoid multi-collinearity between variables in the multivariable linear regression model. POD: postoperative day, TKA: total knee arthroplasty, Exp. Coef: exponentiated regression coefficient, Cl: confidence interval, ASA: American Society of Anesthesiologists.

cally important for TKA [21]. However, since the information regarding the impact of spatial summation in patients with OA on postoperative pain outcome is lacking, more studies should be performed.

There is another important issue regarding central sensitization in this study: preoperative widespread pain. Widespread pain means an expanded distribution of pain; it is a known sign of central sensitization in patients with symptomatic OA [22]. Thus, there is a possibility that the patients who reported pain at other sites may be experiencing central sensitization due to chronic and severe OA. A previous study suggested that widespread pain before TKA is associated with postoperative pain, although it was not a predictor of the amount of pain relief from TKA [23]. Another previous study reported that preoperative widespread hyperalgesia may be a determinant of patients' benefit from TKA [24]. However, the effect of central sensitization on preoperative widespread pain is still unclear, and our findings suggest that this may be associated with an increased morphine equivalent consumption in the postoperative period.
Another interesting finding of the present study is that preoperative analgesic use was not significantly associated with morphine equivalent consumption during POD 0-3. These findings could be explained. Because there are strict regulations regarding opioid misuse or abuse in our country [25], a relatively small proportion of the population are chronic users of opioids for pain not associated with cancer, compared to that in the United States. Specifically, 1.6\% (63/4,007 patients) of the patients in the present study were chronic opioid users preoperatively, compared to $15.7 \%$ (53/338 patients) in an American study population [26]. Considering that preoperative chronic opioid use has been previously found to be an independent factor for the increase of morphine equivalent consumption in the postoperative period [27], the relatively small proportion of chronic opioid users in our cohort may explain why we did not find a similar association in the present study.

There were a few limitations to the study. Firstly, the single center-based retrospective design of the study may result in a lack of generalizability and the possibility of selection bias. 
Table 5. Multivariable Generalized Linear Model for 10 Dependent Variables for Postoperative NRS on POD 0, 1, 2, and 3, and Adjuvant Analgesic Consumption in Entire Cohort $(n=3,429)$

\begin{tabular}{|c|c|c|}
\hline Variable & Exp. Coef $(95 \% \mathrm{Cl})$ & Pvalue \\
\hline \multicolumn{3}{|l|}{ NRS pain score on POD 0: resting } \\
\hline Preoperative knee pain only & 1 & \\
\hline Preoperative knee pain + external knee pain & $1.00(0.93,1.09)$ & 0.944 \\
\hline \multicolumn{3}{|l|}{ NRS pain score on POD 0: moving } \\
\hline Preoperative knee pain only & 1 & \\
\hline Preoperative knee pain + external knee pain & $0.95(0.91,1.02)$ & 0.075 \\
\hline \multicolumn{3}{|l|}{ NRS pain score on POD 1: resting } \\
\hline Preoperative knee pain only & 1 & \\
\hline Preoperative knee pain + external knee pain & $0.98(0.94,1.02)$ & 0.345 \\
\hline \multicolumn{3}{|l|}{ NRS pain score on POD 1: moving } \\
\hline Preoperative knee pain only & 1 & \\
\hline Preoperative knee pain + external knee pain & $0.99(0.95,1.03)$ & 0.658 \\
\hline \multicolumn{3}{|l|}{ NRS pain score on POD 2: resting } \\
\hline Preoperative knee pain only & 1 & \\
\hline Preoperative knee pain + external knee pain & $0.99(0.96,1.02)$ & 0.583 \\
\hline \multicolumn{3}{|l|}{ NRS pain score on POD 2: moving } \\
\hline Preoperative knee pain only & 1 & \\
\hline Preoperative knee pain + external knee pain & $1.00(0.97,1.04)$ & 0.825 \\
\hline \multicolumn{3}{|l|}{ NRS pain score on POD 3: resting } \\
\hline Preoperative knee pain only & 1 & \\
\hline Preoperative knee pain + external knee pain & $0.99(0.96,1.02)$ & 0.473 \\
\hline \multicolumn{3}{|l|}{ NRS pain score on POD 3: moving } \\
\hline Preoperative knee pain only & 1 & \\
\hline Preoperative knee pain + external knee pain & $1.02(0.98,1.06)$ & 0.340 \\
\hline \multicolumn{3}{|l|}{ Acetaminophen consumption (mg) in POD 0-3 } \\
\hline Preoperative knee pain only & 1 & \\
\hline Preoperative knee pain + external knee pain & $0.99(0.95,1.04)$ & 0.795 \\
\hline \multicolumn{3}{|l|}{ Ketorolac consumption (mg) in POD 0-3 } \\
\hline Preoperative knee pain only & 1 & \\
\hline Preoperative knee pain + external knee pain & $0.99(0.95,1.04)$ & 0.795 \\
\hline
\end{tabular}

Backward variable selection method with an elimination criterion of 0.05 was performed to fit the final multivariable GLM for these 10 dependent variables. NRS: numeric rating scale, POD: postoperative day, Exp. Coef: exponentiated regression coefficient, Cl: confidence interval, GLM: generalized linear model.

Secondly, since the prevalence of end-stage OA is higher in female patients and only $9.9 \%$ of the entire study cohort in this study were male patients, it is difficult to generalize our findings to male patients. Third, preoperative NRS pain scores at rest and movement are not routinely assessed at our institution, and only the average NRS pain scores of each patient was evaluated before TKA; therefore, the impact of preoperative pain scores on postoperative pain outcomes was not evaluated in this study, and this may be a limitation. Fourthly, many different registered nurses were involved in assessing the postoperative NRS pain scores over the study period, which may have affected the accuracy of the scores. Finally, the range or number of the external to knee pain sites was not evaluated in this study, and the impact of central sensitization including spatial summation is not clearly demonstrated in this study. Future studies using a prospective design should be performed to focus on this aspect.

In conclusions, this study showed that there was an increased morphine equivalent consumption during POD 0-3 after TKA in patients with preoperative pain in the knee and external to the knee, compared to patients with preoperative pain in the knee only.

\section{CONFLICTS OF INTEREST}

No potential conflict of interest relevant to this article was reported. 


\section{ORCID}

Tak Kyu Oh: https://orcid.org/0000-0002-4027-4423

Chong Bum Chang: https://orcid.org/0000-0002-8597-1054

Hyun-Jung Shin: https://orcid.org/0000-0003-0991-7061

Sunghee Han: https://orcid.org/0000-0002-7058-9952

Sang-Hwan Do: https://orcid.org/0000-0001-5452-4166

Hyun Hee Cho: https://orcid.org/0000-0001-8801-2724

\section{REFERENCES}

1. Maradit Kremers H, Larson DR, Crowson CS, Kremers WK, Washington RE, Steiner CA, et al. Prevalence of total hip and knee replacement in the United States. J Bone Joint Surg Am 2015; 97: 1386-97.

2. Brander V, Stulberg SD. Rehabilitation after hip- and knee-joint replacement. An experience- and evidence-based approach to care. Am J Phys Med Rehabil 2006; 85(11 Suppl): S98-118; quiz S119-23.

3. Beswick AD, Wylde V, Gooberman-Hill R, Blom A, Dieppe P. What proportion of patients report long-term pain after total hip or knee replacement for osteoarthritis? A systematic review of prospective studies in unselected patients. BMJ Open 2012; 2: e000435.

4. Puolakka PA, Rorarius MG, Roviola M, Puolakka TJ, Nordhausen $\mathrm{K}$, Lindgren L. Persistent pain following knee arthroplasty. Eur J Anaesthesiol 2010; 27: 455-60.

5. Teichtahl AJ, Wluka AE, Proietto J, Cicuttini FM. Obesity and the female sex, risk factors for knee osteoarthritis that may be attributable to systemic or local leptin biosynthesis and its cellular effects. Med Hypotheses 2005; 65: 312-5.

6. Imamura M, Imamura ST, Kaziyama HH, Targino RA, Hsing WT, de Souza LP, et al. Impact of nervous system hyperalgesia on pain, disability, and quality of life in patients with knee osteoarthritis: a controlled analysis. Arthritis Rheum 2008; 59: 1424-31.

7. Lee YC, Lu B, Bathon JM, Haythornthwaite JA, Smith MT, Page GG, et al. Pain sensitivity and pain reactivity in osteoarthritis. Arthritis Care Res (Hoboken) 2011; 63: 320-7.

8. Schaible HG. Spinal mechanisms contributing to joint pain. Novartis Found Symp 2004; 260: 4-22; discussion 22-7, 100-4, 277-9.

9. Nie H, Graven-Nielsen T, Arendt-Nielsen L. Spatial and temporal summation of pain evoked by mechanical pressure stimulation. Eur J Pain 2009; 13: 592-9.

10. Staud R, Koo E, Robinson ME, Price DD. Spatial summation of mechanically evoked muscle pain and painful aftersensations in normal subjects and fibromyalgia patients. Pain 2007; 130: 177-
87.

11. Kim MH, Nahm FS, Kim TK, Chang MJ, Do SH. Comparison of postoperative pain in the first and second knee in staged bilateral total knee arthroplasty: clinical evidence of enhanced pain sensitivity after surgical injury. Pain 2014; 155: 22-7.

12. D'Hoore W, Sicotte C, Tilquin C. Risk adjustment in outcome assessment: the Charlson comorbidity index. Methods Inf Med 1993; 32: 382-7.

13. Derby SA. Opioid conversion guidelines for managing adult cancer pain. Am J Nurs 1999; 99: 62-5.

14. Lluch E, Torres R, Nijs J, Van Oosterwijck J. Evidence for central sensitization in patients with osteoarthritis pain: a systematic literature review. Eur J Pain 2014; 18: 1367-75.

15. Fingleton C, Smart K, Moloney N, Fullen BM, Doody C. Pain sensitization in people with knee osteoarthritis: a systematic review and meta-analysis. Osteoarthritis Cartilage 2015; 23: 104356.

16. Rakel BA, Blodgett NP, Bridget Zimmerman M, Logsden-Sackett N, Clark C, Noiseux N, et al. Predictors of postoperative movement and resting pain following total knee replacement. Pain 2012; 153: 2192-203.

17. Staud R, Vierck CJ, Cannon RL, Mauderli AP, Price DD. Abnormal sensitization and temporal summation of second pain (wind-up) in patients with fibromyalgia syndrome. Pain 2001; 91: 165-75.

18. Staud R. Abnormal endogenous pain modulation is a shared characteristic of many chronic pain conditions. Expert Rev Neurother 2012; 12: 577-85.

19. Julien N, Goffaux P, Arsenault P, Marchand S. Widespread pain in fibromyalgia is related to a deficit of endogenous pain inhibition. Pain 2005; 114: 295-302.

20. Ossipov MH, Dussor GO, Porreca F. Central modulation of pain. J Clin Invest 2010; 120: 3779-87.

21. Graven-Nielsen T, Wodehouse T, Langford RM, Arendt-Nielsen L, Kidd BL. Normalization of widespread hyperesthesia and facilitated spatial summation of deep-tissue pain in knee osteoarthritis patients after knee replacement. Arthritis Rheum 2012; 64: 2907-16.

22. Lluch Girbés E, Dueñas L, Barbero M, Falla D, Baert IA, Meeus M, et al. Expanded distribution of pain as a sign of central sensitization in individuals with symptomatic knee osteoarthritis. Phys Ther 2016; 96: 1196-207.

23. Wylde V, Sayers A, Lenguerrand E, Gooberman-Hill R, Pyke $M$, Beswick AD, et al. Preoperative widespread pain sensitization and chronic pain after hip and knee replacement: a cohort analysis. Pain 2015; 156: 47-54.

24. Wylde V, Sayers A, Odutola A, Gooberman-Hill R, Dieppe P, 
Blom AW. Central sensitization as a determinant of patients' benefit from total hip and knee replacement. Eur J Pain 2017; 21: 357-65.

25. Han T. Opioids in cancer and non-cancer pain management in Korea: the past, present and future. Eur J Pain 2001; 5 Suppl A: 73-8.

26. Kim KY, Anoushiravani AA, Chen KK, Roof M, Long WJ, Schwarzkopf R. Preoperative chronic opioid users in total knee arthroplasty-which patients persistently abuse opiates following surgery? J Arthroplasty 2018; 33: 107-12.

27. Armaghani SJ, Lee DS, Bible JE, Archer KR, Shau DN, Kay H, et al. Preoperative opioid use and its association with perioperative opioid demand and postoperative opioid independence in patients undergoing spine surgery. Spine (Phila Pa 1976) 2014; 39 : E1524-30. 